Rout, R.K., et al., Detection of high tritium activity on the central titanium electrode of a plasma focus device. Fusion Technol., 1991. 19: p. 391.

\title{
DETECTION OF HIGH TRITIUM ACTIVITY ON THE CENTRAL TITANIUM ELECTRODE OF A PLASMA FOCUS DEVICE
}

\author{
R. K. ROUT, M. SRINIVASAN, A. SHYAM, and V. CHITRA \\ Bhabha Atomic Research Centre, Neutron Physics Division Trombay \\ Bombay 400 085, India
}

KEYWORDS: plasma focus, cold fusion, tritium measurements

A 2-kJ Mather plasma focus device is used to deuterate the top end surface (or tip) of its central titanium electrode to investigate the occurrence of anomalous nuclear reactions in the context of the "cold fusion" phenomenon. The tip of the central titanium electrode is found to develop at least a few tens of microcuries of tritium after several plasma focus discharges. Neither the tritium impurity level in the deuterium gas used in the experiment nor the tritium branch of the d-d reactions that are known to occur in plasma focus devices can account for such activity in the electrode. Anomalous nuclear reactions in the deuterated titanium lattice appear to be the most probable source of this high activity.

\section{INTRODUCTION}

The occurrence of anomalous nuclear processes in palladium or titanium when loaded with deuterium either electrolytically, ${ }^{1}$ in gas phase, ${ }^{2}$ or in plasma phase ${ }^{3}$ has been reported by various laboratories throughout the world. In gas-phase studies, neutron emission as well as high levels of tritium activity have been observed in two independent Frascati-type absorption-desorption mode experiments with titanium turnings carried out at Bhabha Atomic Research Centre ${ }^{4,5}$ (BARC). Some titanium targets subjected to radiofrequency heating in a $\mathrm{D}_{2}$ atmosphere have shown evidence of tritium activity. ${ }^{6}$ High tritium levels have also been observed in "aged" deuterated titanium targets. ${ }^{7}$ In view of all these results, the same phenomenon was investigated using a plasma focus device ${ }^{8}$ at BARC. Since the tip of the central electrode in a plasma focus device is rapidly heated and cooled in an atmosphere of deuterium gas/plasma and is bombarded with deuterium ions of energy up to $400 \mathrm{keV}$ (Ref. 9), the electrode may be expected to get loaded with deuterium during a discharge shot. This study was carried out to search for the possible occurrence of cold fusion-like anomalous nuclear phenomena in such deuterated electrodes.

\section{PLASMA FOCUS DEVICE AND ITS OPERATION}

A plasma focus device forms a high-density $\left(10^{25} \mathrm{ion} / \mathrm{m}^{3}\right)$, high-temperature $\left(10^{7} \mathrm{~K}\right)$ plasma that produces an intense burst of neutrons when operated with deuterium gas. ${ }^{8}$ This device consists of coaxial cylindrical electrodes separated by an insulator at the 
bottom end and left open at the top, the entire assembly being housed inside a vacuum chamber. Figure 1 shows a schematic of the plasma focus device used in these experiments. The vacuum chamber is $12.7 \mathrm{~cm}$ in diameter and $16.5 \mathrm{~cm}$ long. The outer electrode configuration is a squirrel cage type. The central electrode is $10 \mathrm{~cm}$ long and $2.2 \mathrm{~cm}$ in diameter. Each of the 12 outer electrodes is $11 \mathrm{~cm}$ long and $0.8 \mathrm{~cm}$ in diameter, placed at a distance of $4 \mathrm{~cm}$ from the center of the inner electrode. The Corning glass insulator is $4 \mathrm{~cm}$ long. The plasma focus chamber is typically filled with deuterium gas at a pressure of 100 to $2000 \mathrm{~Pa}$ (1 to 20 mbar).

The device is driven by a $28-\mu \mathrm{F}$ low-inductance $(100 \mathrm{nH}$ at focus formation) fast capacitor bank charged to $12 \mathrm{kV}$ (stored energy of $2 \mathrm{~kJ}$ ). When the spark gap switch is fired, a surface discharge is first initiated along the length of the insulator. This then develops into a radial current sheath that is axially accelerated thereafter by $J \times B$ forces between the electrodes, sweeping and "snow plowing" the gas ahead of it. On reaching the open end, the current sheath turns around on itself, forming a quasi-cylindrical implosion resulting in a dense hot plasma focus a few centimetres long and a few millimetres in diameter, just above the tip of the central electrode.

The time resolved $(d I / d t)$ and voltage were recorded using a Rogowsky coil and a voltage divider, respectively, on separate storage oscilloscopes. The voltage and $d I / d t$ signals indicate whether there is a successful formation of a healthy plasma focus during the discharge.

\section{EXPERIMENT}

The first experiment with a central titanium rod electrode (TA1) was carried out on January 4, 1990. This titanium electrode was exposed to $\sim 50$ charge/discharge shots at different deuterium gas filling pressures with the plasma focus operated in the normal mode, namely with the bank charged to positive voltage $(12 \mathrm{kV})$. Subsequently, 10 shots were taken with the bank charged to negative voltage $(-12 \mathrm{kV})$. The polarity of the voltage was changed by replacing the diode chain with another similar but reverse polarity diode chain in the power supply unit. This was done to deflect some of the highenergy (up to $400 \mathrm{keV}$ but most of the ions have energy 10 to $100 \mathrm{keV}$ ) deuteron beams that are known to be generated in a plasma focus device toward the central electrode. The second titanium electrode (TA2) was subjected to 25 normal shots at a charging voltage of $12 \mathrm{kV}$, while the third electrode (TA6) was given with 25 shots of reversed polarity $(-12 \mathrm{kV})$. A titanium electrode with a central depression (called the hollow titanium electrode) was also used. So far, we have studied four titanium electrodes. Other solid electrodes used were aluminum, brass, stainless steel, and tungsten. 


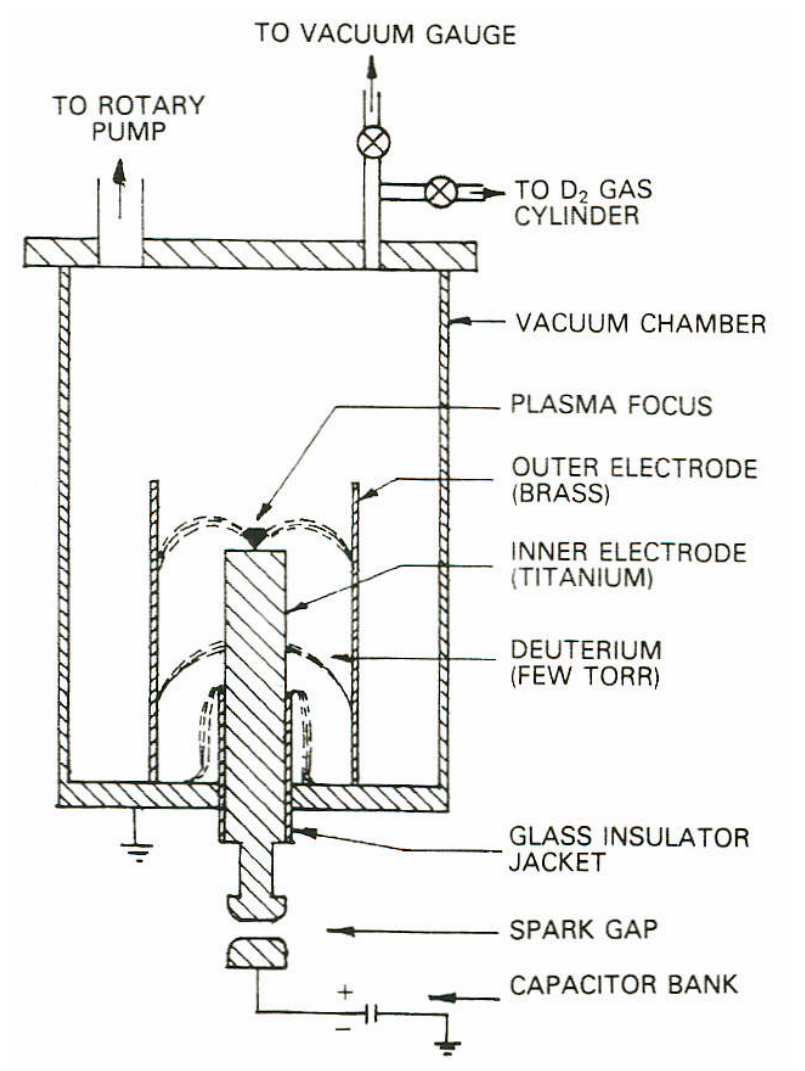

Fig. 1. Schematic of a plasma focus device.

\section{NEUTRON YIELD MEASUREMENT}

The neutron yield depends on the quality of focus formation. In plasma focus devices the neutrons are usually produced in one or possibly two sharp bursts lasting 10 to $100 \mathrm{~ns}$, but the entire emission occurs well within a microsecond. The neutron yield in each shot in this experiment was measured with the help of a set of calibrated activation-type silver cathode Geiger-Muller (GM) counters embedded in paraffin and located close to the device. The GM counters count the 23 -s beta activities induced in its silver cathode by the moderated neutrons. The setup had a threshold sensitivity of $\approx 10^{5} \mathrm{n} /$ burst. For electrode TA1, the neutron yield per shot varied from $5 \times 10^{5}$ to $10^{7}$, depending on the filling pressures.

\section{AUTORADIOGRAPHY}

Autoradiography was the first method employed to look for the presence of radioactivity on the surface of the central electrode. The technique is described in detail in Ref. 6. In this technique, with exposures of several days, localized regions of $\leq 1 \mathrm{~mm}$ (spots) with surface activity levels of a few hundred nanocuries each can be identified. Electrode TA1 was kept on the X-ray film for overnight exposure after the plasma focus shots on January 4, 1990. However, we did not detect any image at that time, presumably because the developer used was defective (this was realized only later). The next imaging was done after 5 weeks, when tritium activity was accidentally discovered on this rod using a newly procured $\mathrm{NaI}$ scintillation detector. When autoradiography was repeated this time, we obtained a spectacular image (see Fig. 2) of the top surface of electrode TA1. The autoradiography of electrodes TA2 and TA6 was done immediately after the plasma focus shots, but these gave only very faint images. Electrodes made 
of other materials and the hollow titanium electrode have, however, failed to give any image so far.

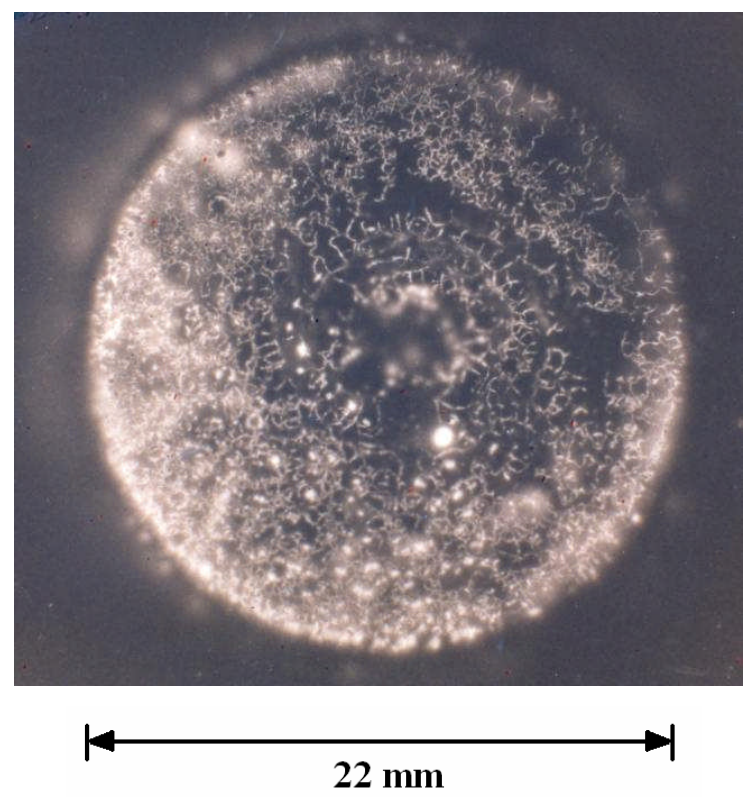

Fig. 2. Autoradiograph of end surface of the central titanium electrode (TA1).

\section{MEASUREMENT OF TRITIUM ACTIVITY}

A 1-mm-thick NaI detector setup with associated electronics feeding into a personal computerbased pulse-height analyzer was used to measure tritium activity. ${ }^{7}$ By sitting on a 6-keV-wide window centered around $5 \mathrm{keV}$, near-surface tritium was measured by means of the Ti-K X rays. Figure 3 shows the X-ray spectrum of electrode TA1 measured by this detector system. The threshold sensitivity of this technique is $\sim 0.2 \mu \mathrm{Ci}\left(\approx 10^{12}\right.$ tritium atoms $)$. Electrode TA1 was measured only 5 weeks after $\mathrm{D}_{2}$ loading, but the other electrodes (TA2 and TA6) were measured immediately after the shots. Table I summarizes the count data for electrodes TA1, TA2, and TA6. A tritiated titanium target of known activity $(76 \pm 8 \mathrm{mCi})$ was used as reference for calibration. The error bar in the activity of tritiated target is due to the uncertainty associated with the measurement of quantity of tritium gas absorbed. The first electrode (TA1) with 50 normal shots and 10 reversed polarity shots has given the highest activity of $\approx 392 \mu \mathrm{Ci}$ (measured 5 weeks after the plasma focus shots). These electrodes were, however, too big to be measured by the available $2 \pi$ beta counter. However, in the case of some deuterated titanium chips, it was observed $^{5}$ that the tritium activity measured by the $2 \pi$ beta counter gave a value that was one or two orders of magnitude less than that measured by the NaI detector. The discrepancy was attributed to differences in the depthwise distribution of tritium between the sample and standard. The second electrode (TA2) with 25 normal shots has given $\approx 8.5 \mu \mathrm{Ci}$, while the third electrode (TA6) with 25 shots of reversed polarity has developed $\approx 2 \mu \mathrm{Ci}$ of activity. This explains why the autoradiographs of electrodes TA2 and TA6 are very faint. The hollow titanium electrode has not shown any activity. 


\section{RESULTS, DISCUSSION, AND CONCLUSIONS}

Neutron yield per shot was $\approx 10^{7}$ on average. But tritium has been observed so far only in the solid titanium electrodes. The tritium measurement by the $\mathrm{NaI}$ detector shows electrode TA1 has produced the highest activity $(\approx 392 \mu \mathrm{Ci})$ among TA1, TA2, and TA6. The deuterium gas used for electrode TA1 was procured from Matheson. Unfortunately, the deuterium in this cylinder was depleted before tritium activity was detected from the plasma focus electrode. Therefore, this gas could not be tested for possible tritium contamination. The deuterium used for TA2, TA6, and for other electrodes are from a different source of supply. The tritium content of the $\mathrm{D}_{2}$ cylinder was measured and found to be $\leq 0.72 \mu \mathrm{Ci} / \ell$. However, this cannot explain the large difference in activity. While the tritium observed in electrodes TA2 and TA6 are of comparable magnitude, it does appear that reverse polarity has not particularly improved tritium production.

The autoradiograph of electrode TA1 (66-h exposure) taken 5 weeks after the plasma focus shots is shown in Fig. 2. This image has been reproduced several times since then through repeated exposures. There has been no noticeable change in the resolution or clarity of the images even after a gap of a few months. The image reveals a large number of randomly distributed spots indicating the presence of localized tritium-bearing sites. Two kinds of structures are discernible in the image. The first type is sharp wormlike lines that appear to have been created by beta particles emanating from the surface. The second variety is diffused spots, which could be attributed to soft $\mathrm{X}$ rays emanating from deeper layers. Attempts are under way to correlate this autoradiograph with the micrograph obtained using an optical microscope with a magnification of over 100.

TABLE I

Measurements on Titanium Rod Electrodes by NaI Detector

\begin{tabular}{|l|l|l|c|}
\hline Electrodes & TA1 & TA2 & TA6 \\
\hline $\begin{array}{l}\text { Date of plasma focus shots } \\
\text { Date of measurement } \\
\text { Count rate with standard }\end{array}$ & $\begin{array}{l}\text { January 4, 1990 } \\
\text { February 21, 1990 }\end{array}$ & $\begin{array}{l}\text { May 24, 1990 } \\
\text { May 24, 1990 }\end{array}$ & June 4, 1990 \\
$76 \pm 8$ mCi source (count/s) & 6,1990 \\
Count rate with electrodes & 6215.7 & 6290.3 & 11268.8 \\
(count/s) & 33.3 & 2.3 & 1.8 \\
Background (count/s) & 1.2 & 1.6 & 1.5 \\
Tritium activity $(\mu \mathrm{Ci})$ & $392 \pm 90$ & $8.5 \pm 1.7$ & $2.0 \pm 0.5$ \\
\hline
\end{tabular}




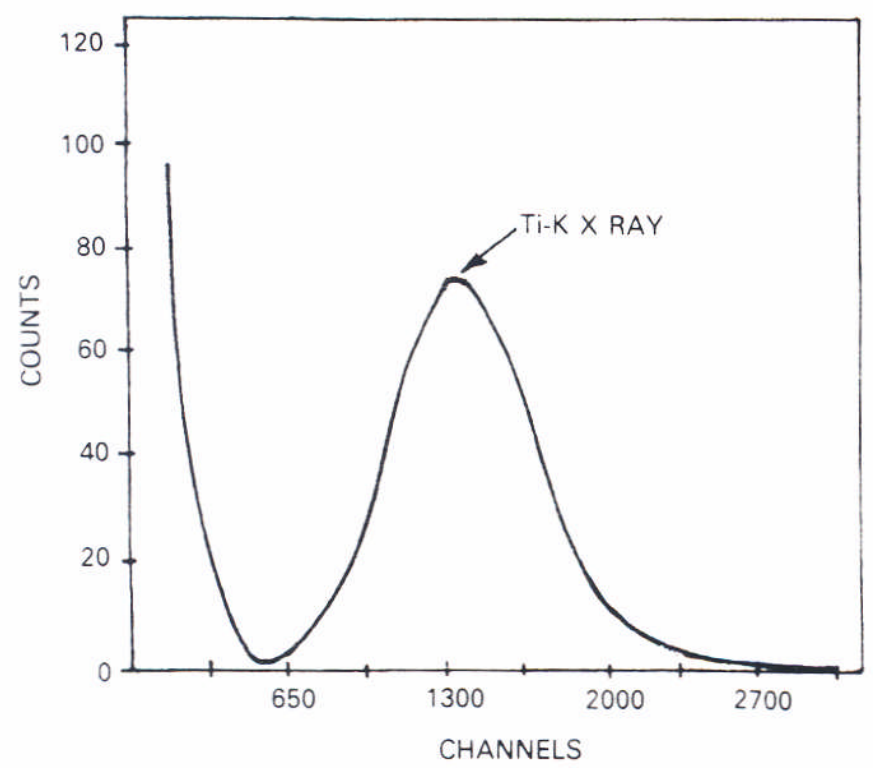

Fig. 3. X-ray spectrum of TA1 measured by a 1-mm-thick NaI detector.

Our 2-kJ plasma focus typically produces $10^{6}$ to $10^{8} \mathrm{n} / \mathrm{shot}$. In the present experiments, assuming 100 shots as the upper limit, not more than $\approx 10^{9}$ neutrons (average neutron yield per shot $\approx 10^{7}$ ) could have been produced in all with each electrode. Since the tritium-to-neutron branching ratio in conventional $d$ - $d$ reactions is unity [the reaction cross sections of $d(d, \mathrm{~T}) p$ and $d(d, \mathrm{He}) n$ are almost equal in a conventional $d-d$ fusion], the same number, $10^{9}$ tritium atoms, would have been produced at most as a result of the hot (or rather beam target) fusion reactions. It is totally unrealistic to expect that all this tritium would have succeeded in getting absorbed in the central titanium electrode. Even if that were true, it still cannot explain the $10^{14}$ (electrode TA2) to $10^{16}$ (electrode TA1) atoms of tritium measured on their tips in this series of experiments.

Since a titanium electrode with a central 2-cm depression did not develop any activity, it appears that the proximity of the surface being exposed to the plasma focus plays an important role in the sequence of events leading to anomalous tritium production.

The temporal variation of neutron yield and tritium activity in these electrodes is proposed to be studied systematically in future experiments. For this purpose, an in situ low-energy beta counter is being fabricated. An on-line personal computer with variable channel width and multiscaling mode of data acquisition is also being installed.

\section{REFERENCES}

1. M. FLEISCHMANN and S. PONS, "Electrochemically Induced Nuclear Fusion of Deuterium," J. Electroanal. Chem., 261, 301 (1989).

2. A. De NINNO et al., "Evidence of Emission of Neutrons from a Titanium Deuterated System," Europhys. Lett., 9, 221 (1989).

3. N. WADA and K. NISHIZAWA, "Nuclear Fusion in Solid," Jpn. J. Appl. Phys., 28, 2017 (1989).

4. P. K. IYENGAR et al., "Bhabha Atomic Research Centre Studies in Cold Fusion," Fusion Technol., 18, 32 (1990). 
5. T. C. KAUSHIK et al., "Preliminary Report on Direct Measurement of Tritium in Liquid

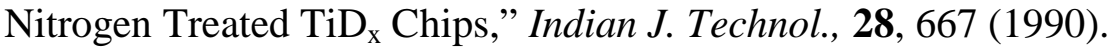

6. R. K. ROUT et al., "Autoradiography of Deuterated Titanium and Palladium Targets for Spatially Resolved Detection of Tritium Produced by Cold Fusion,” Fusion Technol., 18, 83 (1990).

7. M. SRINIVASAN et al., "Observation of High Tritium Levels in Aged Deuterated Titanium Targets as Possible Evidence of Cold Fusion," Fusion Technol., 18, 88 (1990).

8. J. W. MATHER, "Dense Plasma Focus," Meth. Exp. Phys., 9B, 187(1971).

9. H. KROMPHOLZ et al., "Neutron, Ion and Electron Energy Spectra in a 1 KJ Plasma Focus," Appl. Phys., 13, 29 (1977). 